\title{
N92-17996
}

\section{Target Pitch Angle for the Microburst Escape Maneuver}

\author{
Sandeep S. Mulgund \\ Department of Mechanical and Aerospace Engineering \\ Princeton University
}




\title{
Background
}

\author{
- FAA's Windshear Training recommends that upon recognition \\ of encounter with a microburst, pilot should command full thrust \\ and rotate aircraft to an initial target pitch attitude of $15^{\circ}$ \\ - Advantages of the $15^{\circ}$ target: \\ - Easily recalled in emergency situations \\ - Prominently displayed on attitude indicators \\ - It should be possible to postulate a target pitch angle for any \\ airplane \\ - Objective of the present work is to better define the optimum \\ target pitch angle, and develop a methodology for identifying \\ the target pitch for any aircraft
}

The objective of this study was to investigate the constant pitch attitude strategy as a possible non-precision maneuver for recovery from inadvertent wind shear encounters. The Wind Shear Training Aid published by the FAA recommends that upon encountering a severe wind shear, the pilot should apply maximum thrust and rotate the aircraft to an initial pitch target angle of $15^{\circ}$. The $15^{\circ}$ target was identified through rigorous analyses using six-degree-of-freedom flight simulators and microburst models representative of actual accident cases. It was found that $15^{\circ}$ was an effective target for a wide range of shears, and was generally applicable to most jet transports.

This work was undertaken to examine the issue of recovery performance in wind shear of other classes of aircraft - notably turboprop commuters and propeller-driven general aviation planes. It should be possible to postulate a target pitch angle (TPA) for such aircraft, as well. 


\section{Effect of Windshear on Aircraft Performance}

- The impact of a microburst on an aircraft's climb performance is described by the "F-Factor":

$$
F=\frac{\dot{v}}{g} \cdot \frac{w_{h}}{\nabla}
$$

- $F$ is a function of the aircraft's trajectory through the wind shear, and relates directly to its constant speed climb gradient capability

- Maximum trim rate of climb under an imposed F-Factor was computed for a Cessna 402, under two different assumptions:
(a) $F=\frac{\dot{w}}{g}$
(b) $F=-\frac{W_{n}}{V}$

The effect of a wind shear on the climb performance of an airplane is well described the F-Factor, a hazard index developed at NASA Langley Research Center. The F-Factor depends on the time-rate-of-change of the horizontal wind component $\dot{w}_{x}$, the vertical wind component $w h$, the airplane's true airspeed $V$, and the acceleration due to gravity $g$.

An F-Factor of about 0.15 effectively cancels out the climb capability of most transport category aircraft. In an analysis of five wind shear-related accidents, it was found that the aircraft experienced average F-Factors of 0.2 to 0.25 .

To gain insight into the effect of wind shear on climb performance, the maximum constant-speed rate of climb of a general aviation airplane was calculated as a function of an imposed F-Factor. Two different assumptions were made about the source of the F-Factor: first, that it was due entirely to the horizontal shear term, and second, that it was due entirely to the downdraft term. 


\section{Aircraft Model}

- Aerodynamic data taken from simulation model of the Cessna 402B developed at NASA Ames Research Center

- Point mass model of aircraft dynamics

- State variables: $V, h$, and $\gamma$

- Controls are throttle setting and pitch rate

- Climb performance in a wind shear was studied with the aircraft in initial approach configuration: $45^{\circ}$ flaps, gear retracted 


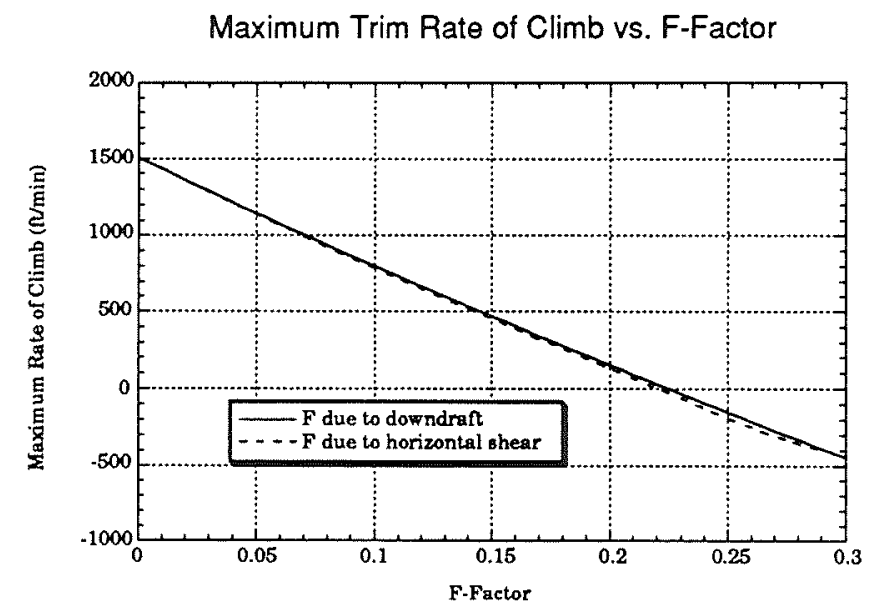

It was found that the maximum trim rate of climb of the airplane decreased almost linearly with the magnitude of $F$. It made little difference whether the F-Factor was due to a downdraft or a horizontal shear. It can be seen from this plot that if $\mathrm{F}$ exceeds 0.22 , the airplane is incapable of maintaining altitude at any constant airspeed. 


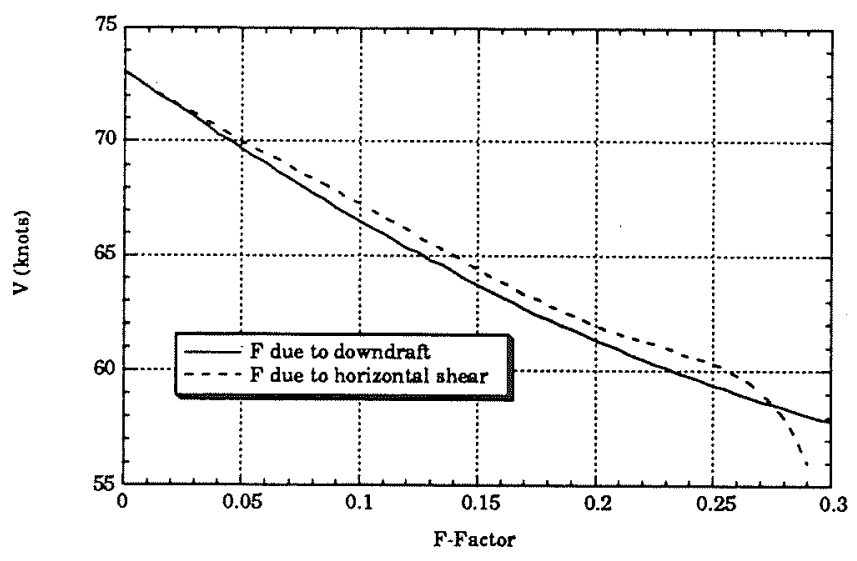

This plot indicates that in a wind shear environment, maximum rates of climb are achieved at airspeeds considerably lower than the "speed for best climb", which is about 73 knots for this airplane model in the approach configuration. This result may be non-intuitive to many pilots, who associate best rate of climb with a specific airspeed. The trend in airspeed for best climb vs. F is the same whether the F-Factor is due to a downdraft or horizontal shear, except at extremely high values of $F$. 


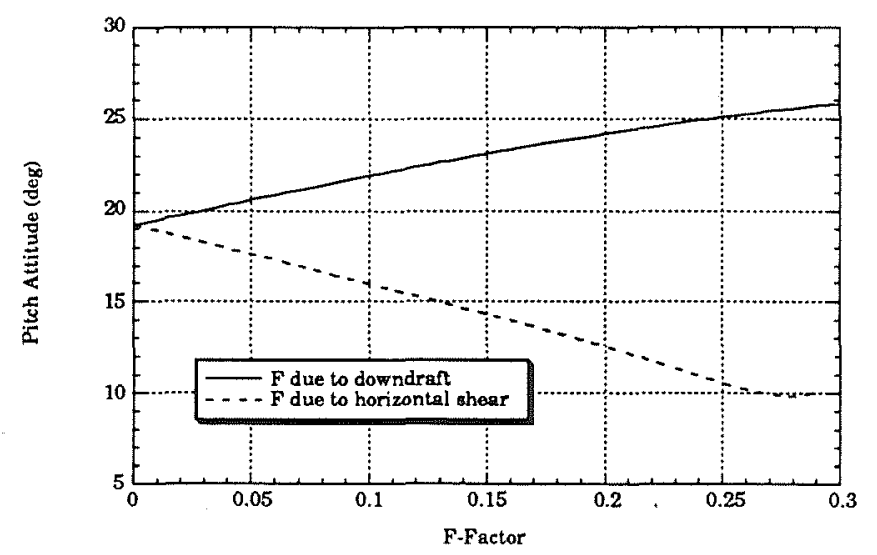

It is here that the difference is seen between the effects of a downdraft and a horizontal shear on the climb performance of the airplane. In the case of a horizontal shear, the greater the F-Factor, the lower the pitch attitude for maximum rate of climb (or minimum rate of descent if $F$ exceeds 0.22 ). Conversely, in a pure downdraft, the greater the F-Factor, the higher the pitch attitude for best rate of climb. This suggests that an effective recovery guidance strategy in a wind shear depends on identifying the source of the threat to the aircraft. In an actual wind shear encounter, an airplane will simultaneously experience horizontal shears and downdrafts.

The objective of this study is to develop a methodology for identifying a single target pitch angle for an airplane which is effective for a reasonable spectrum of possible wind shear encounters. This analysis of climb performance vs. F-Factor suggests that any such target pitch angle will be a compromise between two considerations - In downdrafts, best climb performance is achieved at relatively high pitch attitudes, while in a pure horizontal shear resulting in the same F-Factor hit, best climb performance is achieved at much lower attitudes. 


\section{Validation of the Target Pitch Angle}

- Target pitch angle will be identified for two aircraft:

\begin{tabular}{lrr} 
& MTOW (LB) & Powerplant \\
\hline Cessna 402 & 6,300 & $2 \times 300 \mathrm{HP}$ \\
B737-100 & 93,500 & $2 \times 12,000$ LB
\end{tabular}

- Recovery performance at the target pitch angle will be evaluated by simulating the response of each aircraft to a wind shear generated by an analytic downburst model

The TPA will be identified for two aircraft, representing drastically different weight and powerplant categories. A six-degree of freedom simulation model of the Boeing 737 is currently in use in Princeton's fixed-base simulator. The recovery performance of each aircraft will be simulated using an analytic model of a microburst wind shear. 


\section{Analytic Downburst Model}

- Developed at NASA LaRC by Rosa Oseguera and Roland Bowles.

- Represents an axisymmetric stagnation point flow, based on velocity profiles from the Terminal Area Simulation System Model.

- Time invariant microburst

- Permits simulation of different shears through the specification of three parameters:

1) Radius of the downdraft column

2) Maximum horizontal wind speed

3) Altitude of maximum outtlow

- Wind components in $x, y$, and $z$ directions are easily obtained from the velocity equations for a given aircraft position relative to downburst core. 


\section{Simulation of Microburst Encounters}

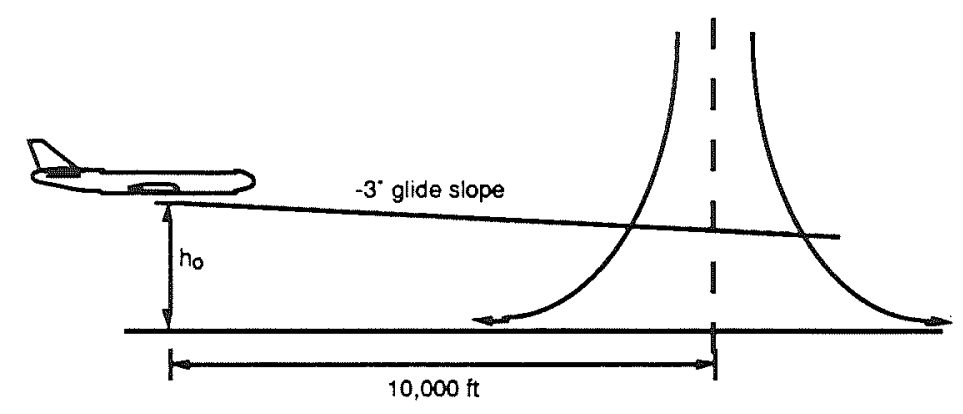

- Aircraft configured on approach along nominal glide slope

- Microburst core placed directly on flight path

Batch simulations of the recovery performance of the Cessna 402 were conducted by initializing the airplane on the glideslope $10,000 \mathrm{ft}$ away from the microburst core. The initial altitude ho was varied during the simulation runs, as was the strength of the microburst. The control logic was set up to track the glide slope until the F-Factor exceeded 0.1, at which point a recovery was initiated by applying full thrust and rotating the aircraft to the TPA. "Stick Shaker" was respected by limiting the angle of attack to $12^{\circ}$. 


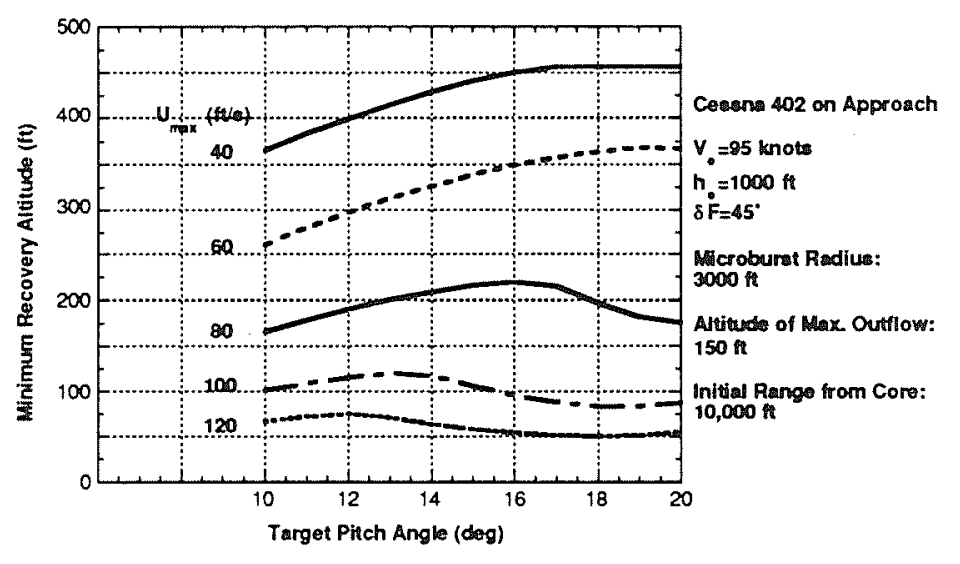

The impact of shear strength was assessed by conducting a series of batch simulations in which the radius of the microburst and the initial location of the airplane relative to its core were kept fixed, and the maximum horizontal wind speed was varied.

The trends in the results suggest that the TPA for maximizing the minimum recovery altitude in a wind shear encounter during approach depends on the strength of the shear. As the maximum horizontal wind speed was increased, it was found that the pitch attitude for maximum recovery altitude decreased. If the target pitch angle was set to one greater than that which maximized recovery altitude, the aircraft was exposed to prolonged periods of stall warning and reduced recovery performance. 


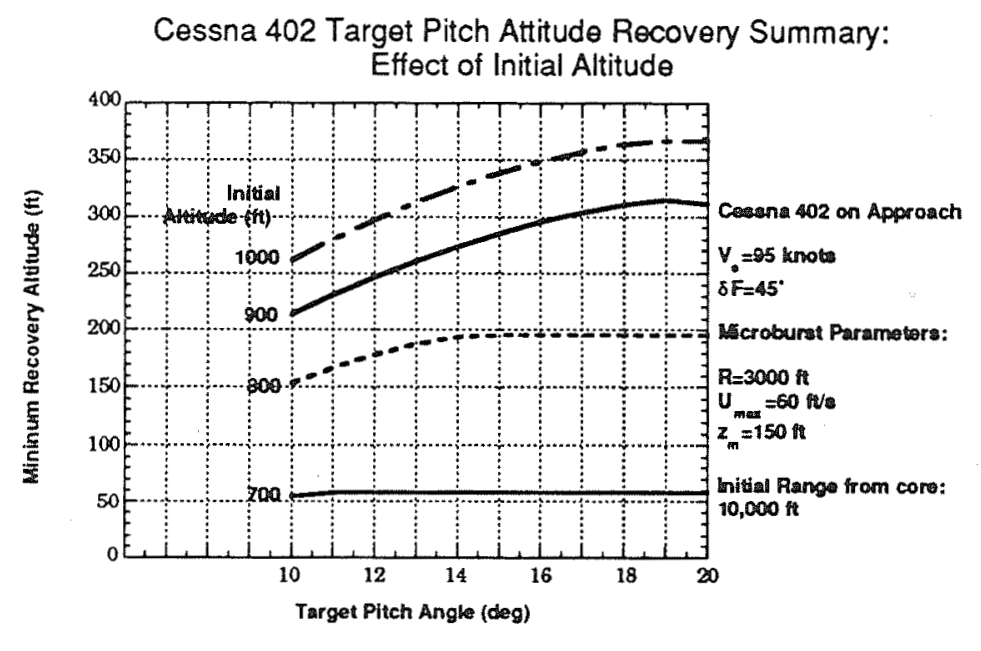

The impact of the altitude at which a wind shear encounter occurs was assessed by fixing the size and strength of the microburst, and varying the initial altitude of the airplane. It was found that as the initial altitude was decreased, so did the target pitch angle that maximized minimum recovery altitude. This result is consistent with those from the analysis of climb performance vs. F-Factor. It was found earlier that in horizontal shears, pitch attitude for best rate of climb decreased with the magnitude of the corresponding F-Factor. Conversely, in downdrafts, pitch attitude for best rate of climb increased with the magnitude of $F$. The simulation results can be interpreted in this context: In low altitude encounters, the aircraft generally encounters "more" horizontal wind than downdraft, simply because vertical winds are smaller nearer to the ground. Consequently, it might be expected that in very low-altitude microburst encounters, best recovery performance is biased toward relatively low target pitch angles. 


\section{Future Work}

- Develop a concise definition of the target pitch angle.

- Evaluate wind shear recovery performance in batch simulations for the two aircraft under consideration.

- Evaluate impact of including pilot in the loop using piloted simulations of wind shear encounters on the LCA Fixed-Base Flight Simulator.

The simulation results of the Cessna 402 model showed what pitch attitudes might be needed for good recovery performance in a wind shear. It was found that there was not one single pitch attitude which produced the best recovery performance in all cases. The target pitch attitude for best recovery depended on the strength of the shear and the altitude at which the encounter took place. It remains to determine what classes of wind shear encounters are most suitable for the definition of the target pitch angle.

There are significant implementation issues associated with the definition of the TPA. For example, can a pilot really distinguish $13.5^{\circ}$ from $15^{\circ}$ ? A typical pitch attitude indicator has markings at $10,12.5,15,17.5$, and $20^{\circ}$. As a practical matter it may be necessary to limit any postulated TPA to one of these values. Also, there is the issue of how well a pilot can maintain any particular pitch attitude in the dynamic environment of a wind shear. In addition to trying to maintain some pitch attitude, a pilot will also be using the ailerons to maintain a near wings-level attitude. These issues will be explored by conducting piloted six-degree-of-freedom simulations in the fixed-base flight simulator in the Laboratory for Control and Automation. Presently, this facility is configured to simulate a Boeing 737-100 transport. 